\title{
OBRA CIENTÍFICA Y HONESTIDAD INTELECTUAL: UN COMENTARIO (Y HOMENAJE) A LA OBRA DE PEDRO PABLO ABREU FUNARI
}

Víctor Revilla Calvo ${ }^{1}$

Como es habitual en la comunidad científica, mi primera aproximación a Pedro Pablo Abreu Funari fue indirecta. Dicho de otro modo, el era para mí lo que solemos denominar un "conocido bibliográfico", alguien a quien conocemos mucho antes por su producción científica que en persona. En el caso de Pedro Pablo, ese conocimiento remonta al periodo, finales de la década de 1980, en que preparaba mi tesis de licenciatura sobre economía romana; seguida años más tarde por una tesis sobre el mismo tema. En ese momento, las indicaciones de mi director de tesis me llevaron a buscar y leer sus trabajos científicos. Esos trabajos me impactaron. No se trataba solo de que la temática se moviera en el ámbito de la economía romana y que afectaran, de algún modo, mi propio trabajo. En ellos había ideas, se proponían hipótesis bien fundamentadas, desarrolladas con un modo de pensar que no era todavía frecuente en la literatura científica española. Detrás había también una metodología poco habitual, determinada por una perspectiva multidisciplinar, que recurría, sin complejos, a la epigrafía sobre el instrumentum, la arqueología y los textos literarios; estos últimos, eran además deconstruidos cuidadosamente, ya que se percibía claramente su condición de imágenes codificadas elaboradas por las elites griegass y romanas.

Este conocimiento indirecto se convirtió en relación personal, con ocasión de una de las varias visitas que Pedro Pablo realizó a España; más concretamente, como resultado de algunas de sus estancias de investigación en nuestra universidad. Fruto de esa relación fue la propuesta de realizar una estancia en la Universidade Estadual de Campinas. Este proyecto fue posible en 1999, gracias a una ayuda de FAPESP. Es difícil resumir las impresiones de esa estancia, determinante

\footnotetext{
1 Profesor Titular, Universitat de Barcelona, Barcelona, España. E-mail: victorrevillac@gmail.com
} 
para mí, a pesar de no ser muy larga, tanto a nivel científico como personal. Gracias a esa estancia tuve oportunidad de conocer otra comunidad académica, otra práctica científica $\mathrm{y}$, sobre todo, otra sociedad, la brasileña, construida sobre la base del multiculturalismo. Llegué a Brasil algo desorientado, sin nada más que tópicos sobre el país, que muy pronto me mostró toda su diversidad. También algo desorientado sobre qué podría aportar al plan de estudios de una universidad como Campinas. ¿Podía interesar una investigación centrada en la historia de la conquista y la economía de Hispania? Y sobre todo ¿esa investigación podía conectar intelectualmente con las necesidades personales de unos estudiantes para los cuales la Historia de Roma no constituía un legado cultural acríticamente aceptado?

Desde luego, el balance fue extremadamente positivo para mí. En Campinas encontré otra forma de organizar y de sentir la vida universitaria, dentro de un marco académico que tenía poco que ver con el de la universidad española: un marco de convivencia y actividad científica construido a partir de una relación más cercana y una mayor libertad en la relación profesor-alumno, el intercambio de opiniones y la vocación; todo ello construido a partir de la constatación de la fuerte diversidad de procedencias e intereses de los miembros de la comunidad universitaria. Tuve ocasión, igualmente, de vivir y conocer otras situaciones (en Sao Paulo, en Joinville), en el ámbito universitario y en la vida cultural de los museos locales y regionales, cosa que me permitió apreciar las motivaciones que explican la relación entre sociedad, ciencia y patrimonio que ha inspirado la acción y el pensamiento de Pedro Pablo. Mi estancia en Brasil también me sirvió para conocer a algunos de sus alumnos y discípulos. La relación con ellos ha continuado en años posteriores, gracias a estancias en la Universidad de Barcelona. Todos ellos muestran la misma motivación, libertad de pensamiento y curiosidad científica de su maestro. Algo que puede definirse como la "marca de fábrica" de una escuela.

Este breve resumen de circunstancias personales me sirve, a modo de introducción, para realizar un comentario más específico sobre lo que ha significado la influencia de la obra de Pedro Pablo en diversos ámbitos de la historiografía y la arqueología de la Antigüedad Clásica en España. 
La obra de Pedro Pablo Funari se ha desarrollado en ámbitos realmente muy diversos, desde los estudios de cultura material de la antigüedad más especializada hasta la arqueología histórica, seguramente una de sus áreas de trabajo preferidas. En el caso de algunos investigadores esta diversificación corre el peligro de conducir a la dispersión y a la superficialidad. En el caso de Pedro Pablo, este rasgo es el reflejo de una profunda curiosidad intelectual que tiene efectos positivos ya que le ha permitido aprovechar repetidamente las ideas y las prácticas mentales desarrolladas en un área de investigación a otros ámbitos. Bastará con analizar el impacto del trabajo del autor en algunos campos de alta especialización.

El primero, el de la cultura material romana, donde el autor ha dedicado una atención especial a las evidencias arqueológicas relacionadas con la producción y exportación de productos alimentarios en la antigua Roma; en particular, al estudio de la tipología y la epigrafía de las ánforas romanas como documento que permite la cuantificación, la datación y la cartografía de los flujos comerciales. Este estudio tiene la ventaja añadida de que ofrece la posibilidad de profundizar en el conocimiento de la sociedad romana al analizar los fenómenos de consumo y gusto desarrollados por las diversas clases o colectivos sociales. En las últimas décadas, a partir de 1960-1970, los estudios dedicados a la cultura material de la antigüedad y, en particular, a sus producciones cerámicas, han experimentado un desarrollo acelerado. Este fenómeno se ha traducido en una auténtica explosión de publicaciones que, con diversa extensión y profundidad, abarcan todas las posibilidades de análisis: desde las monografías de excavación, en las que se describen los repertorios asociados a las formas de vida y trabajo de un asentamiento, hasta las síntesis regionales que abordan cuestiones relacionadas con los mecanismos económicos, culturales y políticos que impulsaron la creación de unos circuitos de intercambio de alcance mediterráneo. Más recientemente, a partir de la década de 1990, al compás del desarrollo de la informática, se han introducido en Historia Antigua, nuevos instrumentos de recogida, catalogación y análisis de la documentación que han permitido crear bases de datos cada vez más amplias y complejas. Un ejemplo de ello son, entre otras, la base de datos sobre epigrafía anfórica dirigida por J. Remesal desde la Universidad de Barcelona, online desde 1991 (http://ceipac.ub.edu), o la base sobre 
tipología y arqueometría anfórica impulsada por S. J. Keay en la Universidad de Southampton (Roman Amphorae: a Digital Resource, desde 2005) ${ }^{2}$. Estas bases de datos, que integran documentación tradicional (tipologías, epigrafía) y de nuevo tipo (arqueometría), han proporcionado un impulso renovado al estudio de la cultura material. Por ejemplo, permitiendo establecer relaciones entre diversos tipos de datos $o$ secuencias cronológicas, así como el análisis estadístico y la cuantificación. Esta última posibilidad es imprescindible en historia económica, como se aprecia en el intenso debate teórico generado en los últimos años (Bowman \& Wilson, 2009; Callataÿ, 2014).

Este interés creciente por la cerámica refleja la conciencia de la importancia de este tipo de documentación para plantear cuestiones de carácter histórico; baste citar, como ejemplo, el problema que supone definir, en términos de continuidad e innovación, los cambios que se producen en las estructuras sociales o económicas y las mentalidades entre la República y el Imperio, para lo cual la cultura material aporta evidencias importantes en forma de tradiciones artesanales(locales, regionales, provinciales) o de nuevos hábitos de consumo y de trabajo (es el caso del fenómeno del reciclaje en el ámbito doméstico). Los nuevos interrogantes, en clave histórica, que plantea esta evidencia material tan modesta han generado una renovación total de perspectivas y de planteamientos de trabajo.

Los nuevos procedimientos analíticos, en particular, han permitido superar el recurso exclusivo a la clasificación tipológica como forma tradicional para identificar las producciones y reconstruir los circuitos de intercambio. De forma necesaria, esta renovación ha conducido a la definición de espacios de debate cada vez más acotados por los problemas tratados y los métodos utilizados. Siguiendo la experiencia desarrollada en el estudio de la cultura material de otros periodos, los distintos tipos de cerámicas (especialmente, las vajillas de mesa y las

\footnotetext{
2 Pueden citarse otros recursos igualmente importantes, aunque con una intención más limitada: como la base de datos sobre ánforas de fabricación hispana del Institut Català d'Arqueologia Clàssica de Catalunya (http://amphorae.icac.cat) o la dedicada a la arqueometría de las ánforas galas de los siglos I a III d.C. gestionada por la Maison Archéologie \& Ethnologie, René-Ginouvès de Nanterre (www.mae.uparis10.fr/terresdamphores)
} 
ánforas) son tratados cada vez más como objetos de estudio específicos que requieren una aplicación particular de los procedimientos analíticos generales y la elaboración de hipótesis apropiadas. Sólo de esta forma puede alcanzarse un mejor conocimiento de las relaciones con otras manufacturas (entendiéndolas globalmente como resultado de unas tradiciones artesanales y culturales) y con la producción de otros bienes para, en última instancia, lograr un conocimiento más amplio de ciertos aspectos de la economía y de las sociedades antiguas. Toda la obra de Pedro Pablo Funari se integra en ese contexto de renovación teórica y metodológica, superando la perspectiva centrada en la clasificación tipológica de categorías particulares de vajilla o de las ánforas. Es fundamental, al respecto, la monografía redactada con C. Carreras sobre la circulación del aceite de Baetica o Africa Proconsularis en las provincias septentrionales del imperio romano: Britannia y el Mediterráneo. Estudios sobre el abastecimiento de aceite bético y africano en Britannia (Barcelona, 1998).

Dedicar una monografía al estudio de la distribución y el consumo de un producto como el aceite no es resultado de una elección caprichosa y ello por varias por varias razones. En primer lugar, porque el aceite es, con el vino y los cereales, uno de los componentes de la famosa tríada mediterránea. Su cultivo se asocia a una ecología específica y a unas condiciones de producción que las sociedades protohistóricas e históricas del mundo oriental y mediterráneo desarrollaron y perfeccionaron a lo largo de milenios. Se trata, en otras palabras, de un recurso que simboliza un sistema socioeconómico y es, a la vez, un factor asociado a procesos de cambio y contacto cultural. El aceite es, por otro lado, un producto con usos muy diversos: alimento y condimento, combustible para iluminación, componente en la higiene, la cosmética o la, medicina, etc. $\mathrm{Su}$ uso, en gran cantidad y de forma regular, se asocia a un standard de vida propiamente romano y depende, en las provincias septentrionales del imperio estudiadas por Pedro Pablo, de la existencia de estructuras de comercialización. Pero este uso también depende de la difusión y aceptación (o no) de ciertos hábitos de consumo que suponen cambios significativos en las prácticas culinarias. Muestra de ello son las diferentes formas de consumo en asentamientos civiles y militares de Britania y las Germanias que Pedro Pablo ha sabido tener en cuenta. En esta percepción 
se adivina el interés por la historia de la cultura y las identidades culturales que constituye otro de sus ámbitos de trabajo. El estudio de su difusión, por tanto, ha sido un medio de profundizar en el conocimiento del impacto de la conquista romana sobre las sociedades celtas y germanas que ocupaban el espacio del continente europeo entre los siglos I a.C. y II-III d.C. Los trabajos de Pedro Pablo, insertos en un diálogo enriquecedor con la obra de otros investigadores (por ejemplo: Remesal, 1986 y 1997; Whittacker, 1989), han contribuido a reconstruir las dinámicas históricas generadas por la conquista romana en el centro y occidente de Europa, situando la economía en su justo lugar. En la misma perspectiva se integran otras obras, como Cultura material e arqueología historica (Campinas, 1998) y, sobre todo, Mercato. Le commerce dans les mondes grec et romain (Paris, 2012). Esta obra, escrita en colaboración con uno de los máximos especialistas sobre finanzas y banca en el mundo romano, Jean Andreau, contiene una selección de textos literarios clásicos que son analizados con profundo rigor.

Otro de los ámbitos de interés a los que Pedro Pablo ha concedido interés es lo que se ha denominado comúnmente la Historia de las mentalidades, cuya perspectiva es diferente a la de una Historia de la Cultura (y todavía más respecto a la de una simple Historia de las culturas). No hace falta insistir en como los discursos literarios de los autores antiguos, miembros de una élite que pensaba, actuaba y se imaginaba a escala imperial, ofrecen una imagen del orden social basada en la aceptación de una jerarquía natural, con diferencias igualmente naturales de riqueza, estatus y condición jurídica; por no hablar de las desigualdades apoyadas en el género y la edad, que convierten a algunos colectivos en invisibles; esta invisibilidad afecta en especial a la mujer, los grupos de edad (los ancianos o los niños) y los esclavos. El estudio de la identidad cultural es una perspectiva que pretende dar voz a otros grupos, marginados por su género, condición, estatus, pertenencia cultural, etc., rompiendo con el mensaje unidireccional, hegemónico y de autorepresentación, de las élites de una sociedad. La producción científica de Pedro Pablo, imposible de resumir aquí, es un ejemplo de una sensibilidad científica nueva, que supone el desarrollo de nuevas perspectivas, pero también de una metodología diferente, que analiza evidencias nuevas o ya conocidas. 
El ámbito más evidente es el de los graffiti. Esta práctica es particularmente importante en el mundo romano, como muestran las inscripciones parietales de Pompeya y otros lugares de la Campania, así como los millares de grabados sobre cerámica recuperados en todo el imperio. A través de esta evidencia, que ha impactado fuertemente a la comunidad científica desde su descubrimiento en el siglo XVIII y que afectó al modo tradicional (a la vez, académico y convencional) de valorar la sociedad romana, ha sido posible aproximarse de modo diferente a los valores y los comportamientos de colectividades marginadas en el registro literario, definir los procesos que conducen a la creación de formas de identidad colectiva e individual (social, cultural, de género, etc) y analizar las relaciones y conflictos entre los diversos grupos que integra una sociedad tan fuertemente jerarquizada como la romana; una sociedad en la que, como resultado de la conquista romana del mediterráneo, confluyen una cultura de élite (determinada por un lenguaje estético y unos modelos éticos helénicos profundamente readaptados en el periodo helenístico) y un mosaico de culturas regionales muy diversas. Estas culturas, a su vez, habían sido más o menos influidas por el legado griego desde época arcaica: púnicos, etruscos, celtas, egipcios, persas. Estudiar las manifestaciones escritas de la sociedad romana, más allá de la literatura culta, significa desarrollar una doble perspectiva y una doble sensibilidad, a la vez social y cultural. Sólo así es posible intentar desentrañar los rasgos que caracterizan las diversas culturas locales en el imperio o la cultura popular romana (de límites imprecisos de definir) y su evolución en el tiempo. De modo más concreto, solo desde esta sensibilidad se puede percibir el proceso de creación cultural a través de las cuales diversos colectivos definieron su identidad y se situaron en un contexto histórico complejo. El imperio romano, con su extensión y diversidad, es, en ese sentido, una realidad muy compleja y difícil de captar.

En este contexto histórico complejo, el uso de la escritura en formas diversas, desde el signo a la palabra, en combinación con el uso de imágenes, debió contribuir a dar nuevos significados a los objetos cotidianos sobre los que se escribieron, ya que les atribuía un valor comunicativo que relacionaba individuos y ciertas iniciativas, que se concentraban en un lugar. Esto es lo que sucede con los millares de 
objetos inscritos que ha recuperado la arqueología, redactados tanto en latín como en las otras lenguas que incluía el imperio. Pero esta auténtica invasión de la palabra escrita y de la imagen se aprecia también en otro tipo de soportes: las paredes.

Este conjunto de prácticas de comunicación, se desarrollaron en lugares y situaciones precisas, en relación con unas intenciones o unas funciones que no siempre se pueden precisar o que no se explicitan claramente. Son mensajes cotidianos e inmediatos, que responden a necesidades de expresión inmediata y directa; en otras palabras, son mensajes codificados, cotidianos, sin pretensión de eternidad, difíciles de desentrañar. Son, precisamente por estas razones, un medio fundamental de aproximarse a las creencias y los comportamientos reales de gran parte de la población del imperio, ya que son parte de mecanismos de comunicación social y de definición de identidades desarrollados por individuos, pequeños colectivos y comunidades en diversos escenarios y situaciones. Entre estas se puede considerar la religión, pero también los complejos juegos de alusiones y de identificación personal generados por la convivencia y el trabajo cotidiano que aparecen en la paredes o sobre vajilla. Ninguna de estas posibilidades se puede excluir. Como han puesto de relieve diversos investigadores, la definición de identidades en la sociedad romana implicaba procesos activos de selección entre un repertorio de imágenes y una cultura material, en continua redefinición, generada dentro de un marco imperial; esta selección se acompañaba de un uso preciso de diversas formas de mensaje escrito (Alfayé \& Marco, 2008; Woolf, 1995; Hingley, 2005).

Es en este campo de estudio donde se integra una obra de pequeño formato, breve y sencilla, pero muy importante: La cultura popular en la antigüedad clásica (Écija, 1991). En ella se recoge e interpreta un conjunto de ejemplos de grafitos parietales de Pompeya caracterizados por la combinación de texto e imagen. Todos ellos se conocen desde antiguo y numerosos investigadores han destacado, justamente, los temas que tratan estos grafitos: situaciones relacionadas con el ambiente de los juegos gladiatorios (combates, héroes populares), el sexo, los chismes de vecindario o de la vida local. El análisis que se hace de estas evidencias en este libro ha sabido destacar algunos principios que caracterizan el 
lenguaje popular y sus códigos: los juegos de palabras y las alusiones más o menos claras, las exageraciones humorísticas, el uso cuidadoso de los insultos o la palabra soez (no siempre ofensivos) y del doble sentido. También se ha destacado otro aspecto fundamental: la ya aludida combinación de texto e imagen. Esta combinación es más profunda de lo que puede parecer, ya que el texto no funciona simplemente como didascalía informativa. El texto adquiere también un valor estético, supera los límites tradicionales de la ordinatio, convertido en otra imagen. De este modo, una frase asume una configuración sinuosa o una forma que sugiere el mensaje transmitido: la fealdad de una persona a través de rasgos somáticos esquematizados; la potencia sexual, etc. La disposición del texto, en resumen, refuerza y completa, todo cuanto se representa con la imagen. Son elementos inescindibles de un mismo mensaje. El análisis de Pedro Pablo, a través de ejemplos muy bien escogidos, profundiza en esta y otras ideas para descubrir un auténtico código. Igualmente importante es el análisis de la estructura interna de los textos y los elementos componentes, organizados en torno a una serie. Muchos de esos aspectos se amplían en trabajos posteriores ${ }^{3}$.

La influencia de esta monografía se aprecia en la obra de otros autores, como N. Horsfall (1996 y 2003), y puede percibirse en trabajos muy recientes, como la monografía de Kristina Milnor (2014) dedicada al "Literary Landscape in Roman Pompeii". Se podrá argumentar que los grafitos parietales de las ciudades del Vesubio solo permiten una aproximación parcial y sesgada al conocimiento de la cultura de las clases inferiores de la sociedad romana (una visión "italocéntrica"). Pero hay suficientes indicios de la extensión de esta práctica en todo el Mediterráneo, en todo tipo de situaciones y sobre todo tipo de soportes materiales (cerámica, paredes, piedra) para utilizar la evidencia de Pompeya y Herculano como el medio más directo para aproximarse a las realidades y preocupaciones cotidianas de una sociedad de dimensiones imperiales. Utilizando una vieja y afortunada expresión, también en esta esfera el periodo imperial supone una extensión del "epigraphic habit". El trabajo de Pedro Pablo se ha completado con la explotación de líneas

\footnotetext{
3 P. P. A. Funari, "El carácter popular de la caricatura pompeyana", Gerión, 11, 1993, 153-173, especialmente pp. 169 y siguientes. También "Graphic caricature and the ethos of ordinary people at Pompeii", Journal of European Archaeology, 1, 1993, 133-150.
} 
de investigación que abordan otras temáticas y recurren a otros filones documentales. Se puede citar, al respecto, Aspectos de La Cultura Popular Romana a Partir de Pompeya. Arte, erotismo y sensibilidad en el mundo romano (Madrid, 2012). Varios de los discípulos de Pedro Pablo han desarrollado esta línea de investigación con gran provecho. Basta recordar algunas de las monografías de Renata Senna Garraffoni (Bandidos e salteadores na Roma antiga. Concepçoes da elite romana sobre a transgressao social, Sao Paulo, 2002; Gladiadores na Roma antiga, Sao Paulo, 2005) o la obra de Lurdes Feitosa (Amor e sexualidade. O Masculino e o Feminino em Grafites de Pompéia, Sao Paulo, 2005).

Paralelamente, el trabajo de Pedro Pablo se inserta en un campo de estudios más amplio y que ha conocido un particular desarrollo en las últimas décadas: el cambio cultural y los procesos de definición de las identidades en diversos ámbitos: social, religioso, político. El debate al respecto es muy importante y ha iniciado con el cuestionamiento radical de perspectivas (la perspectiva colonial, en primer lugar) y de la misma terminología, empezando por la palabra "romanización". La bibliografía al respecto es enorme y el debate es muy animado (Woolf 1992, 1995, 1997a-b y 1998; Webster, 2001; Hingley 2005; Witscher 2000; Terrenato 1998, 2008 y 2013). En este escenario, los estudios pioneros sobre cultura popular, un término en sí mismo difícil de definir adquieren un nuevo valor por la importancia que conceden al estudio integral de los grafitos como combinación específica de texto, imagen y soporte como un medio de definir las identidades individuales y colectivas en la sociedad romana (Cooley 2002; para la cultura popular en la Roma imperial: Horsfall 2003; Toner 2009). El análisis del autor se ha ampliado en los últimos años a la perspectiva de la identidad, como muestran algunas obras importantes y coloquios editados: Identidades, discurso e poder. Estudos da arqueología contemporânea (Sao Paulo 2005), Politica e identidades no mondo antigo (Sao Paulo, 2009), Identidades fluídas no judaísmo antigo e no cristianismo primiivo (Sao Paulo, 2010). El tema de la identidad es analizado en estos trabajos desde perspectivas muy diversas (sociedad, religión, guerra, política) que relacionan mundo antiguo y nuestra época contemporánea.

Esto nos conduce hasta otro ámbito de estudio, de gran actualidad y que sobrepasa los límites estrictos de la investigación académica: la 
"recepción" del mundo clásico. El interés por los problemas en torno a la transmisión del legado clásico, su contribución a la definición de una identidad (europea u occidental) o su manipulación responden, a mi juicio, a una idea particular: la "recepción" como un diálogo, en continua revisión, entre nuestra sociedad moderna y el pasado. Este diálogo se convierte en debate cuando se evalúa el peso del legado clásico en la composición de la identidad de la sociedad brasileña contemporánea La percepción de la actualidad de este debate inspira iniciativas como la obra New Perspectives on the Ancient World: Modern perceptions, ancient representations (British Archaeological Repports International Series núm. 1782, Oxford, 2008), editada por Pedro Pablo junto a R. S. Garraffoni y B. Letalien.

A su vez, la actividad de Pedro Pablo en este campo inspira la elaboración de material didáctico y pedagógico, buena parte del cual he conocido y utilizado directamente. Su actividad se ha materializado en la preparación de instrumentos de trabajo de tipo muy diverso. Estos instrumentos se han pensado para su utilización en contextos muy diferentes, con actores igualmente diversos: el gran público, el aficionado deseoso de profundizar en el mundo antiguo, los jóvenes a punto de acceder a la universidad. La elaboración de este tipo de instrumentos es especialmente difícil, ya que se debe evitar por igual la simplificación (que implica reducir una información a unas pocas fórmulas reductivas) y la homogenización excesiva. Este doble proceso de simplificación y homogenización encierra otro peligro: construir una imagen del pasado idealizada y que reproduce del discurso elaborado por unas élites, sea la sociedad esclavista romana sea el Brasil moderno y contemporáneo, una sociedad también parcialmente basada en la esclavitud. Sobre este aspecto ya se ha insistido anteriormente. La obra de P.P. Funari evita este peligro y es consciente de la necesidad de dirigirse a personas y colectivos cuyos intereses y necesidades evolucionan en función del contexto cotidiano en el que actúan, como aficionados, como escolares, como "consumidores" de cultura, etc.; los mismos individuos, pero con intereses cambiantes. Afortunadamente, la comunidad científica es cada vez más consciente de la obligación ética de difundir los resultados de una actividad investigadora que ha sido sostenida con una financiación pública y de revestir esos resultados a la sociedad. Una obligación ética, 
desde luego, pero también una actuación guiada por factores prácticos: buscar el apoyo social para una práctica científica cada vez más costosa y cada vez más difícil de justificar por la simple curiosidad cientíifca. La obra de Pedro Pablo es pionera en ese sentido, no solo en la sociedad brasileña, sino también por su influencia en el trabajo de muchos colegas en otros países del continente americano. Esta obra recoge una amplia gama de matices: Arqueologia (Sao Paulo, 1988), A Cidade e a civilização romana: um instrumento didático (Campinas, 1997), Antigüidade clássica: a história e a cultura a partir dos documentos (Campinas, 2003), Patrimônio histórico e cultural y Patrimônio e cultura material (ambos publicados en 2006), Arqueologia e patrimonio (Erechim, 2007).

Es imposible hacer justicia al valor de la obra de Pedro Pablo Funari o, más sencillamente, intentar resumir su impacto en el ámbito de las ciencias históricas; sea en Brasil, en el ámbito académico americano o en España. Si hubiera que explicar en que reside este valor, habría que hacer referencia sin duda a la combinación de rigor científico, honestidad intelectual y sensibilidad. Una combinación rara en nuestra profesión que he tenido el privilegio de conocer personalmente.

\section{Bibliografia}

ALFAYÉ, S.; MARCO, F. Religion, language and identity in Hispania: Celtiberian and Lusitanian rock inscriptions. In: HÄUSSLER (ed.). Romanisation et épigraphie. Études interdisciplinaires sur l'acculturation et l'identité dans l'Empire romain. Montagnac: Éditions Monique Mergoil, 2008, 281-305.

BOWMAN, A. K. \& WILSON, A. I. (eds.). Quantifying the Roman Economy: Methods and Problems. Oxford: Oxford University Press, 2009.

CALLATAY, F. de (ed.). Quantifying the Greco-Roman Economy and Beyond, Bari: Edipuglia, 2014.

CARRERAS MONFORT, C.; FUNARI, P.P.A. Britannia y el Mediterráneo: estudios sobre el abastecimiento de aceite bético y africano en Britannia. Barcelona: Edicions Universitat Barcelona, 1998.

COOLEY, A. (ed.). Becoming Roman, Writing Latin? Literacy and Epigraphy in the Roman West. Portsmouth, RI: Journal of Roman Archaeology, 2002. 
FEITOSA, L.C. Amor e sexualidade: o masculino e o feminino em grafites de Pompeia. São Paulo: Annablume, 2005.

FUNARI, P.P.A. Arqueologia. São Paulo: Ática, 1988.

La cultura popular en la Antiguedad Clásica. Écija: Editorial Sol, 1991. 1993a, 153-173.

Graphic caricature and the ethos of ordinary people at Pompeii, Journal of European Archaeology, 1, 1993b, 133-150.

A cidade e a civilização romana: um instrumento didático. Campinas: Universidade Estadual de Campinas, Instituto de Filosofia e Ciências Humanas, 1997.

Cultura material e arqueologia histórica. Campinas: Universidade Estadual de Campinas, Instituto de Filosofia e Ciências Humanas, 1998.

Antiguidade clássica: a história e a cultura a partir dos documentos. Campinas: Unicamp, 2003.

Patrimônio e Cultura Material. Campinas: Universidade Estadual de Campinas, Instituto de Filosofia e Ciências Humanas, 2006.

. Arqueologia e patrimônio. Erechim: Habilis, 2007.

Aspectos de la cultura popular romana a partir de Pompeya. Arte, erotismo y sensibilidad en el mundo romano. Barcelona: Editorial Académica Española, 2012.

FUNARI, P.P.A.; ORSER JR, C.E.; SCHIAVETTO, S.N.O. Identidades, discurso e poder: estudos da arqueologia contemporânea. São Paulo: Annablume, 2005.

FUNARI, P.P.A.; PELEGRINI, S. Patrimônio Histórico e Cultural. Rio de Janeiro: Jorge Zahar Editor, 2006.

FUNARI, P.P.A.; GARRAFFONI, R.S.; LETALIEN, B.L. (eds.). New perspectives on the ancient world: modern perceptions, ancient representations. Oxford: Archaeopress, 2008.

FUNARI, P.P.A.; SILVA, M.A.O. (eds.). Política e identidades no mundo antigo. São Paulo: Annablume/Fapesp, 2009. 
FUNARI, P.P.A.; NOGUEIRA, P.A. de S.; COLLINS, J.J. (org.). Identidades fluidas no judaísmo antigo e no cristianismo primitivo. São Paulo: Annablume/Fapesp, 2010.

GARRAFFONI, R.S. Bandidos e salteadores na Roma antiga. São Paulo: Annablume/FAPESP, 2002.

. Gladiadores na Roma antiga: dos combates às paixões cotidianas. São Paulo: Annablume, 2005.

HINGLEY, R. Globalizing Roman Culture: unity, diversity and empire, Londres: Routledge, 2005.

HORSFALL, N. M. La cultura della plebs romana, Barcelona: Promociones y Publicaciones Universitarias, 1996.

HORSFALL, N. M. 2003: The Culture of the Roman Plebs, Londres: Bristol Classical Press, 2003.

MATTINLGY, D. J. Imperialism, Power, and Identity. Experiencing the Roman Empire, Princeton: Princeton University Press, 2011.

MILNOR, K. Graffiti and the Literary Landscape in Roman Pompeii. Oxford: Oxford University Press, 2014.

REMESAL RODRÍGUEZ, J. La annona militaris y la exportación de aceite de oliva en Germania. Madrid: Editorial de la Universidad Complutense, 1986.

REMESAL RODRÍGUEZ, J. Heeresversorgung und die wirtschaftlichen Beziehungen zwischen der Baetica und Germanien. Materialien zu einem Corpus der in Deutschland veröffentlichten Stempel auf Amphoren der Form Dressel 20. Stuttgart: Kommissionsverlag Konrad Theiss, 1997.

TERRENATO, N. The Romanization of Italy: global acculturation of cultural bricolage. In: FORCEY, C., HAWTHORNE, J., WITCHER, R. (eds.). TRAC 97. Proceedings of the Seventh Annual Theoretical Roman Archaeology Conference. Oxford: Oxbow Books, 1998, 20-27.

TERRENATO, N. The cultural implications of the Roman conquest. In: BISPHAM, E. (ed.). Roman Europe (the short Oxford history of Europe). Oxford: Oxford University Press, 2008, 234-264.

TERRENATO, N. Patterns of cultural change in Roman Italy. Non elitereligion and the defense of cultural self-subsistency. JEHNE, M., LINKE, B., RÜPKE, J. (eds.). Religiöse Vielfalt und soziale Integration. Die Bedeutung 
der Religion für die kulturelle identität und politische Stabilität im republikanischen Italien. Heidelberg: Verlag Antike, 2013, 43-60.

WEBSTER, J. Creolizing the roman provinces, American Journal of Archaeology, 105/2, 2001, 209-225.

WHITTAKER, C. R. Les frontières de l'Empire Romain. Besançon: Université de Franche-Comté, 1989.

WITCHER, R. Globalisation and roman identity: perspectives on identities in roman Italy. In: HERRING, E., LOMAS, K. (eds.). The Emergence of State Identities in Italy in the First Millennium BC. Londres: Accordia Research Institute, University of London, 2000, 213-225.

WOOLF, G. The unity and diversity of romanization, Journal of Roman Archaeology, 5, 1992, 349-352.

WOOLF, G. The Formation of Roman Provincial Cultures. In: METZLER, J. et alii (eds.). Integration in the Early Roman West. The role of Culture and Ideology. Luxemburgo: Musée National d'Histoire et d'Art, 1995, 9-18.

WOOLF, G. Beyond Romans and natives, World Archaeology 28/3, 1997, 339-350.

WOOLF, G. Becoming Roman: The Origins of Provincial Civilization in Gaul. Cambridge: Cambridge University Press, 1998. 